\title{
Methodologies for driving ambient systems
}

\author{
Haroon Malik ${ }^{1}$ Ansar-Ul-Haque Yasar $^{2} \cdot$ Elhadi M. Shakshuki $^{3}$
}

Published online: 28 January 2017

(C) Springer-Verlag Berlin Heidelberg 2017

This special issue is based on selected papers from the Seventh International Conference on Ambient Systems, Networks and Technologies (ANT), which was held at Madrid, Spain on 23-26 May 2016. The conference attracted a large number of scientific papers that contributed to the state-ofthe-art in the areas of pervasive and ambient information systems including social networks, wireless sensor networks, authenticity and internet-of-things. All the papers selected for this special issue has been extended from their original versions and underwent two rounds of rigorous peer-review process. Based on the reviewers' feedback, as well as the evaluations of the guest editors, seven papers were accepted for this special issue from ten invited and submitted papers. The accepted papers cover interesting works on new developments in ambient and networked systems such as internet-of-things, avionics systems, wireless sensors network, and social network.

The first paper by Caprio et al. is titled "The Emergence of Inclusive and Exclusive Virtual Communities Determined by the Preferences of their Users". In social network sites (SNs), users are always faced with the challenge of

Elhadi M. Shakshuki

elhadi.shakshuki@acadiau.ca

Haroon Malik

malikh@marshall.edu

Ansar-Ul-Haque Yasar

ansar.yasar@uhasselt.be

1 Division of Computer Science, Marshall University, Huntington, WV, USA

2 Transportation Research Institute Hasselt University, Hasselt, Belgium

3 Jodrey School of Computer Science Acadia University, Wolfville, Canada accepting or rejecting a friendship request when there is incomplete information about the main characteristics and preferences describing the requester. Towards this end, the paper has introduced a novel decision theoretical model of friendship acceptance, with incomplete information, that has been used to build different network structures determined by the different expectations and preferences of its users. The proposed model has the capacity to deal with ambiguous environments and complement many existing models.

The second paper by Eltayesh et al. is entitled "Refined Game-Theoretic Approach to Improve Authenticity of Outsourced Databases". The authors of this paper aimed to extend their previous work on single follower Stackelberg VDB model. Their previous model only considers one attacker type whereas the approach in this paper proposed Bayesian Stackelberg model that allows the inclusion of multiple attacker types. This feature enables the verifier to obtain a mixed strategy that is optimized with respect to all attacker types' interests and their probability of being faced in the game. Test results showed the proposed model was significantly faster and required less number of verifications to detect manipulations originating from multiple attacker types. The authors analyzed the need and importance of verification moments' randomization and discussed the optimal randomization method to accomplish it. A detailed discussion on the implementation settings of the various model components is presented.

The third paper by Robati et al. is entitled "Design and Simulation of Distributed IMA Architectures using TTEthernet: A Model-Driven Approach". Avionics are the electronic systems used on aircraft, artificial satellites, and spacecraft. Avionic systems include communications, navigation, the display and management of multiple systems, and the hundreds of systems that are fitted to aircraft 
to perform individual functions. Avionic systems based on integrated Modular Avionics (IMA) architecture interconnected with Time-Triggered Ethernet (TTEthernet) have to meet strict real-time safety requirements. In order to overcome the complexity of such systems, the paper advocate a model-based approach, which provides the systems engineers with a methodology and the supporting tools, to accomplish correctly and efficiently, the safe integration of data traffics with different performance reliability requirements.

The fourth paper by Djammaa et al. is entitled "Hybrid CoAP-based Resource Discovery for the Internet of Things". The emergence of Internet of Things (IoT) has led to the development of new routing protocols such as RPL (Ipv6 routing protocol of low-power and lossy networking (LLN)) and MPL (IPv6 Multicast Protocol of LLNs). Such standards have enabled smart physical-world devices to communicate in the Internet. In order to fully exploit the potential of the LLN network stack into providing successful adoptions of IoT, seamless and automatic discovery of available resources is an imperative. This article introduces efficient, scalable and adaptive hybrid centralized/ distributed discover architecture of CoAP resources. The proposed architecture builds upon two main mechanisms, namely proactive resource directories discovery and fully distributed push-pull resource discovery, allowing it to ensure efficient, scalable, and quick discovery of available resources. This paper provided an analysis of the time/cost performance of the proposed architecture followed by an extensive experimental evaluation.

The fifth paper by Sheltami et al. is entitled "Performance Comparison of Three Localization Protocols in WSN using COOJA". The paper evaluates the accuracy of three known localization protocols namely: fingerprint, centroid and DV-Hop using Tmote sky in Cooja; a Contiki network simulator that facilitates the simulation of Contiki motes in small and large networks. The findings of the paper confirms to the theory that fingerprint protocol has better performance than centroid and DV-Hop protocols in terms of localization accuracy. Whereas, DV-hop and centroid protocols outperform fingerprint protocol in terms of stability and power consumption.

The sixth paper by Al-Ayyoub et al. is entitled "Feature Extraction and Selection for Arabic Tweets Authorship
Authentication". Designing systems to automatically verify the true authors of user-generated content (i.e., author authentication over the social-networks such as Twitter can be of great importance). Especially for addressing many problems in forensics, market manipulation, and online shopping reputation systems. Towards this end, the paper proposes two approaches, i.e., 'Bag-of-Words' and 'Stylometic Feature' to address the AA problem. The paper mainly focuses on the tweets containing Arabic languages. The authors created different sets of features from both approaches and compared the performance of different classifiers including Naïve Bays, Decision Trees and Support Vector Machines (SVM). The results showed that combing all feature set yields the best results. They also highlighted the superiority of the SVM classifier over the other classifiers.

The seventh paper by Syed et al. is entitled "A new Traffic Route Analyzer for Commuter's Guidance in Developing Countries: Application study in Islamabad, Pakistan”. This paper provided an approach to facilitate the commuter to pick the route that reduces the hurdles and minimize the distance to reach the destination in less time. The authors proposed solution targeted the traffic system in the city of Islamabad, Pakistan. The authors main aim is to overcome the load of traffic by providing remote facilities in the hands of the commuter to calculate the real time and the distance travelled between source and destination using apps through android. In this paper, the authors evaluated different approaches to optimize the calculated data to provide better solution for the difficult traffic flow for this specific region. The proposed traffic route analyses system based on artificial neural network scheme indicates the hurdles for each route and provided the shortest route analyses based on severity levels.

The guest editors would like to take this opportunity to thank all the authors for the efforts they put in the preparation of their manuscripts and for their valuable contributions. We wish to express our deepest gratitude to the referees who provided very useful and thoughtful feedback to our authors. Our sincere thanks go to the Editor-in-Chief for his kind help and support. 